\title{
Los Grandes Almacenes de Autoservicio Ante la Globalización: El Caso de las Tiendas del Corporativo Wal Mart de Jalisco
}

\author{
Rosario Cota Yáñez ${ }^{(*)}$
} Brenda Cota Yánez ${ }^{(*)}$

\begin{abstract}
Resumen: Las ventas de las tiendas de autoservicios en México se han incrementado a pesar de las crisis económicas. Las tiendas de autoservicio han aprovechado las oportunidades ofrecidas por el mercado. Tomando en consideración estrategias relacionadas con la teoría de la empresa, esto les ha permitido sacar ventajas y lograr mejores posiciones en un mercado oligopolico inestable. Mediante información suficiente del mercado y alianzas estratégicas, como algunas de las seguidas por los líderes del mercado de tiendas de autoservicio en La Zona metropolitana de Guadalajara (ZMG). Específicamente el caso de Wal Mart.
\end{abstract}

Palabras-clave: Grandes Almacenes, Comercio, Wal Mart, México, Globalización.

Abstract: Supermarket sales have increased in Mexico in spite of the economic crises, as selfservice stores have taken advantage of the opportunities that were provided by the market. By taking into account strategies related to business theory Wall Mart has made gains and improved its position in an unstable oligopoly market structure by implementing policies which include holding sufficient information on the market and strategic alliances, such as those between leading supermarkets in the Metropolitan Zone of Guadalajara.

Keywords: Supermarkets, Trade, Wal Mart, Mexico, Globalization.

\footnotetext{
(*) Profesora-investigadora de la Universidad de Guadalajara, Doctora en Ciencias Sociales, Departamento de Estudios Regionales DER-INESER. E-mail: macotaya@cucea.udg.mx. Se agradece el apoyo de la estudiante de licenciatura Elisa Jazmín Martín Gómez.

(**) Estudiante de la Maestría en Negocios y Estudios Económicos, Universidad de Guadalajara. Becaria del Consejo Nacional de Ciencia y Tecnología (CONACYT).E-mail: becy1525@hotmail.com. Recebido em 2.4.09 e aceito em 29.4.09.
} 


\section{INTRODUCCIÓN}

La modernización comercial coexiste a su vez con el mercado tradicional de la metrópoli, pero últimamente está enfrentando cambios, debido entre otras cosas, a las preferencias del consumidor. El objetivo del presente trabajo es analizar el comportamiento de las tiendas Wal Mart en la ZMG y de que forma coexiste el comercio tradicional (mercados públicos, tianguis). La hipótesis se centra en el hecho de que la proliferación de las grandes cadenas de autoservicios están cambiando los patrones de consumo de la población debido a la competencia oligopolica que realizan. Ofrecen mejores precios, calidad, accesibilidad, horarios amplios. Lo anterior se ha visto reflejado en el cierre de pequeños negocios que repercute en la economía de las familias, y en la creación de infraestructura urbana adecuada a las necesidades de este tipo de establecimientos que requieren amplios estacionamientos.

La Zona Metropolitana de Guadalajara ${ }^{(1)}$ ha sufrido una serie de modificaciones con el transcurso del tiempo. El sector comercial es un eje articulador de su dinámica. La ciudad se ha caracterizado por ser el epicentro de intercambio mercantil hacia la región occidente y noroeste del país.

Dos factores son representativos. El primero está relacionado con cambios en la estructura interna como consecuencia del crecimiento urbano. Situación que ha ocasionado el proceso de conurbación. El segundo es la localización comercial que atiende a las fuerzas del mercado, lo cual ha propiciado desequilibrios en la distribución de los centros de abastecimiento de bienes de consumo básico.

El objetivo del presente trabajo consiste en analizar la transformación económica que está sufriendo actualmente la zona metropolitana de Guadalajara (ZMG) ante la introducción de las cadenas comerciales de tiendas de autoservicio que están acaparando el mercado de la región.

La hipótesis es que con la aparición de los supermercados y centros comerciales, la población ha modificado sus patrones de compra. Los centros comerciales han permitido que los compradores no tengan que transportarse a diferentes lugares para adquirir las mercancías necesarias. Como una respuesta se han construido plazas comerciales que responden a las necesidades concretas de los vecinos.

El documento se encuentra dividido en cuatro partes: en la primera se va a hablar sobre la importancia de la actividad comercial en la zona metropolitana de Guadalajara, posteriormente, de las tiendas de autoservicio el caso de Wal Mart; el tercer apartado se refiere al contexto actual del mercado de tiendas de autoservicio en la ZMG, para finalizar con el comportamiento estratégico de Wal Mart.

\section{Antecedentes}

En Jalisco se observan tres etapas dentro del desarrollo comercial. La primera se caracterizó por la participación del capital local y estuvo presente hasta la década de los sesentas. En la segunda etapa se observa la incorporación del capital nacional a finales de la década de los años ochentas, para dar cabida posteriormente a la introducción del capital internacional a mediados de los años 90's.

(1) Comprende los municipios de Tlaquepaque, Tonalá, Zapopan, Tlajomulco, y El Salto 
En 1945 aparece la primera abarrotera en Guadalajara, se caracterizó por ser una tienda que ofrecía carnes, artículos para el hogar y productos frescos. Todo se encontraba en un solo espacio y tenía un horario amplio. Todavía en la década de los 50's existía un comportamiento del comercio tradicional que acudía a los mercados municipales y tianguis de la ciudad. Este tipo de comercios se localizaba en los barrios de la ciudad, cuya función se centraba en el abastecimiento de productos de forma continua.

Es hasta 1962 cuando aparecen los primeros supermercados en la ciudad, se diferenciaron de las abarroteras porque ofrecían mejores precios por medio de la estrategia de los descuentos ${ }^{(2)}$ además, de contar con la venta de ropa, línea blanca, ferretería y tlapalería. Se les conoció como Maxi y su competencia Hemuna ${ }^{(3)}$.

El comercio en Guadalajara se ajustaba y respondía a la tendencia mundial que se estaba gestando en ese tiempo con la aparición de centros comerciales ${ }^{(4)}$.

Durante la década de los setentas Las plazas comerciales y supermercados se ubicaron principalmente en los fraccionamientos y zonas de altos ingresos.

A finales de la década de los setentas encuentran su consolidación los supermercados por el hecho de que las costumbres y hábitos de trabajo, su ubicación se dio principalmente hacia el poniente de la ciudad en los municipios de Guadalajara y Zapopan principalmente. Debido a la necesidad de incorporar nuevos horarios y formas de abastecimiento a diferencia de los mercados tradicionales que sólo abrían por la mañana. La población podía hacer sus compras de manera semanal. El gobierno por su parte, contribuye en esta dinámica mediante la instalación de tiendas como Conasupo ${ }^{(5)}$, Tiendas del Instituto de Seguridad Social al Servicio de los Trabajadores del Estado - ISSSTE ${ }^{(6)}$ y Tiendas del Instituto Mexicano de Seguridad Social - IMSS.

En el segundo quinquenio de la década de los ochentas se inicia un nuevo periodo dentro de la dinámica comercial en los supermercados en Guadalajara se inicia la introducción del capital nacional y posteriormente el internacional. Mediante la venta de los espacios comerciales de tiendas de origen local a las cadenas nacionales ${ }^{(\tau)}$.

A partir de los años 90's se observa dentro de la estructura urbana la incorporación de los denominados megamercados o hipermercados, estilo americano, que abarca la venta de todo tipo de productos, y que además albergan dentro de los mismos comercios o servicios como puede ser el caso de ópticas, comida rápida entre otros.

Los centros comerciales son construidos por el colectivo social de dos formas: como lugares para adquirir mercancías y centros de esparcimiento. Ya que dentro de los mismos se han instalado restaurantes, discotecas, cafés y salas cinematográficas.

(2) Debido a que los almacenes podían adquirir mercancía por grandes volúmenes, les permitía negociar precios con sus proveedores.

(3) El formato comercial seguido por Maxi antecedió a los centros comerciales actuales. Alrededor del supermercado se contaba con locales comerciales para renta de negocios de diferente giro.

(4) A finales de la década de los sesenta se observa un nuevo modelo comercial en Guadalajara con el surgimiento del centro comercial denominado "Plaza del Sol", primer centro comercial de Latinoamérica. Posteriormente se implementaron como modelo al desarrollarse otros complejos como "Plaza Patria" y "Plaza México". A finales de la década de los noventas se presenta una aceleración con la construcción de "Plaza Pabellón", "Plaza Galerías", "Centro Magno", "Plaza Camichines" y las "Plazas Outlet".

(5) Estaba enfocada principalmente a la población de bajos ingresos, se ubicaban hacia el oriente de la ciudad. Actualmente este tipo de tiendas no existen, su desaparición se dio al cierre del Consejo Nacional de Consumo Popular

(6) Este formato de tiendas estaba dirigido a trabajadores adscritos al gobierno del federal con precios bajos. Actualmente siguen prestando sus servicios a todo el público.

(7) "Hemuna" y "Maxi", venden sus locales a la cadena de supermercados Gigante y expande su cobertura mediante la ubicación de las tiendas ya posicionadas. 
Pese a lo anterior, el comercio en pequeña escala sigue funcionando en los barrios de la ciudad. Ya que son negocios familiares que cumplen una función específica dentro de la economía del hogar. Son abastecedoras de los productos que a diario consumen. El reto para estos se presenta a partir del año 2000 con la agresiva incorporación de las denominadas

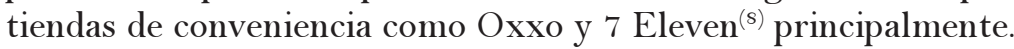

La localización es un factor de éxito o fracaso comercial. Actualmente en la ciudad de Guadalajara existen dos modelos de comercialización de productos perecederos: Los tradicionales y los novedosos.

Dentro de los tradicionales figuran los mercados municipales, los tianguis, las centrales de abasto y los abarrotes (tiendas de la esquina). Dentro de los novedosos están las tiendas de autoservicio también denominadas hipermercados (Wal Mart, Gigante, Soriana, Comercial Mexicana, Chedraui). La comercialización al mayoreo por medio de cadenas (Sam’s Club, Price Club, Bodega Aurrerá), Las plazas comerciales que han incorporado diversos espacios en un sólo lugar comida rápida, tiendas ancla, las tiendas de conveniencia (Oxxo, Seven Eleven, am pm).

\section{Importancia de la ACTIVIDAd COMERCial en la Zona Metropolitana de Guadalajara}

La función económica urbana que antaño había desempeñado Guadalajara desde los años 40 's presenta un resquebrajamiento para dar cabida al desarrollo y apertura de servicios especializados vinculados a la producción y desconcentración de actividades manufactureras, dando lugar a la incorporación de empresas de capital transnacional (POZOS PONCE, 2004, p. 138; RODRÍGUEZ BAUTISTA, 2005).

La Zona Metropolitana de Guadalajara en los últimos 20 años ha presenciado una transformación paulatina pero sostenida en su base económica. Pasó de ser una ciudad especializada en la actividad industrial a tener una fuerte presencia de trabajadores en las actividades del sector terciario (Gráfica 1). Aunque el comercio no presenta un ritmo de crecimiento acelerado, ha logrado mantener su preeminencia junto con los servicios.

\section{Gráfica 1}

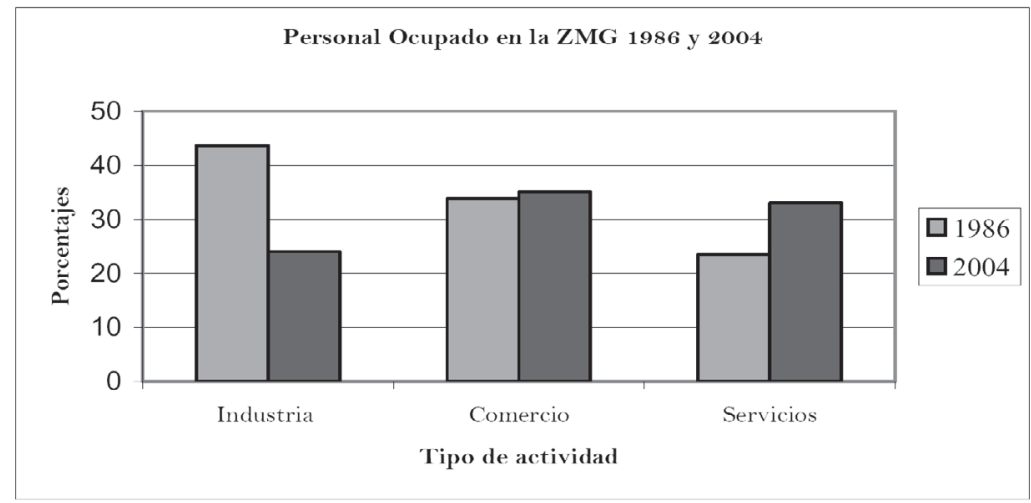

FUENTE: Elaboración propia con base en los Censos Económicos, 1986 y 2004. Instituto Nacional de Estadística Geografía e Informática.

(8) Este tipo de tiendas maneja un nuevo formato al tradicional de los abarrotes. Su estrategia se basa en ofrecer una imagen moderna, limpia, amplios horarios (24 horas). Se maneja mediante concesiones familiares. 
La actividad industrial empieza su proceso de declinación en 1994, aunque todavía mantenía su predominio, es hasta el año 2004 cuando se puede decir que ya no acapara a la población ocupada, los habitantes de la Zona Metropolitana de Guadalajara han optado por la opción de las empresas de comercio y servicio.

En cuanto al sector servicios se observa un incremento progresivo en los servicios distributivos, por la llegada de grandes empresas de autoservicio como Wal Mart y la expansión de otras como Soriana, Gigante y Chedraui que desplazaron en cierta medida a pequeños establecimientos comerciales de capital local.

La tendencia es la expansión de las actividades comerciales mediante nuevos formatos de venta, bodegas, megamercados y plazas comerciales. Los servicios distributivos ${ }^{(9)}$ y personales ${ }^{(10)}$ presentan un aumento en su participación del valor agregado como resultado el crecimiento del comercio al por mayor y al por menor mediante el formato de los megamercados y las tiendas de autoservicio, tiendas de conveniencia (Oxxo, Seven Eleven) y restaurantes (Cuadro 1).

Cuadro 1 - Participación porcentual del personal y el valor agregado por tipo de actividades en la ZMG 1998-2004

\begin{tabular}{|l|r|r|r|r|}
\hline \multicolumn{1}{|c|}{ Subsector } & \multicolumn{2}{|c|}{ Personal } & \multicolumn{2}{c|}{ Valor agregado } \\
\hline & 1988 & 2004 & 1988 & 2004 \\
\hline Productos básicos & 24.7 & 10.5 & 23.3 & 18.8 \\
\hline Intermedios & 12.8 & 7.5 & 19.2 & 10.1 \\
\hline De capital & 5.1 & 7.4 & 11.2 & 8.2 \\
\hline Total manufactura & $\mathbf{4 2 . 6}$ & $\mathbf{2 5 . 4}$ & $\mathbf{5 3 . 7}$ & $\mathbf{3 7 . 1}$ \\
\hline Servicios distributivos & 33.2 & 38.5 & 31.6 & 38.3 \\
\hline Productivos & 4.2 & 13.8 & 3.5 & 13.7 \\
\hline Sociales & 5.2 & 8.2 & 2.6 & 5.3 \\
\hline Personales & 14.9 & 13.4 & 8.6 & 5.2 \\
\hline Total servicios & $\mathbf{5 7 . 5}$ & $\mathbf{7 3 . 9}$ & $\mathbf{4 6 . 3}$ & $\mathbf{6 2 . 5}$ \\
\hline Total & 100.0 & 100.0 & 100.0 & 100.0 \\
\hline
\end{tabular}

FUENTE: Elaboración propia con base en los Censos Económicos, 1986 y 2004. Instituto Nacional de Estadística Geografía e Informática.

Jalisco empieza a tener un repunte en cuanto a la inversión extranjera directa en la década de los 90's (Gráfica 2) con el nuevo boom que presentó la industria electrónica y la participación del sector comercial. La inversión extranjera directa en el 2004 se dirigió hacia el Distrito federal, Estado de México, Nuevo León y Jalisco. Provino principalmente

(9) Comprende a la actividad comercial y del transporte.

(10) Servicios personales, de esparcimiento, restaurantes y hoteles. 
de países como Suiza, Estados Unidos y Japón de acuerdo a datos del Sistema Estatal de Información Jalisco (SEIJAL, 2007).

\section{Gráfica 2}

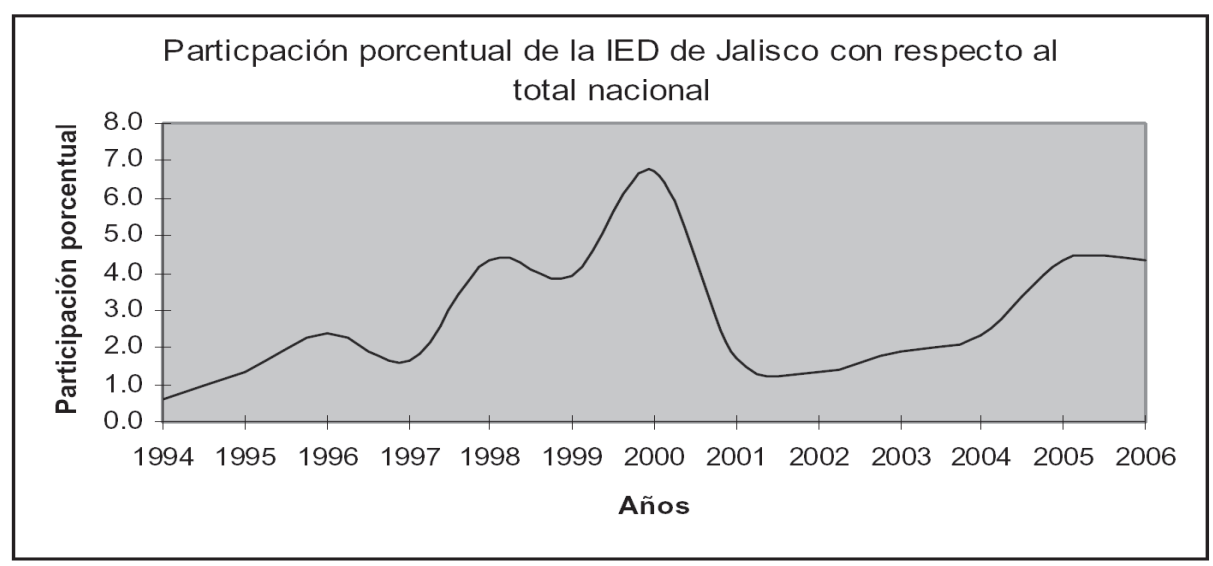

FUENTE: Elaboración propia con base en la Secretaría de Economía. Dirección General de Inversión Extranjera.

La preeminencia del los servicios distributivos se ve reforzada por la participación porcentual de la inversión extrajera directa en Jalisco, que en los últimos años se ha enfocado hacia tres actividades principalmente: la industria manufacturera, actividades financieras y el comercio (Cuadro 2). La inversión en la manufactura se dirige hacia municipios fuera de la ZMG.

\section{Cuadro 2 - Participación porcentual de la inversión extranjera directa en Jalisco por sectores}

\begin{tabular}{|c|c|c|c|c|c|c|c|c|}
\hline Sectores & 1999 & 2000 & 2001 & 2002 & 2003 & 2004 & 2005 & 2006 \\
\hline TOTAL & 100.0 & 100.0 & 100.0 & 100.0 & 100.0 & 100.0 & 100.0 & 100.0 \\
\hline Agropecuario & 0.5 & 0.0 & 1.3 & 0.6 & 0.6 & 0.0 & 0.0 & 0.0 \\
\hline Minería & 2.2 & 2.2 & 4.5 & 1.4 & 1.2 & -2.4 & 0.6 & 0.8 \\
\hline Industria manufacturera & 82.2 & 69.1 & 86.6 & 79.0 & 69.9 & 85.6 & 74.2 & 90.6 \\
\hline Electricidad y agua & 0.0 & 0.0 & 0.0 & 0.0 & 0.0 & 0.1 & 0.0 & 0.0 \\
\hline Construcción & 0.4 & 0.0 & 0.0 & 0.0 & 0.0 & 0.1 & 0.1 & -0.1 \\
\hline Comercio & 7.1 & 5.3 & 8.2 & 10.3 & 12.9 & -3.3 & 6.0 & 0.7 \\
\hline Transportes y comunicaciones & 0.0 & 2.0 & 3.5 & 0.3 & -0.1 & -0.1 & 0.0 & 0.0 \\
\hline Servicios financieros & 0.1 & 0.6 & 4.1 & 15.0 & -2.7 & 8.5 & 2.0 & 2.1 \\
\hline Otros servicios ${ }^{(1)}$ & 7.6 & 20.9 & -8.2 & -6.6 & 18.2 & 11.6 & 17.1 & 5.8 \\
\hline
\end{tabular}

FUENTE: Secretaría de Economía. Dirección General de Inversión Extranjera. 
Dentro de la industria manufacturera sobresale la presencia de la fabricación y ensamble de maquinaria, equipo y accesorios eléctricos, elaboración de productos de plástico, y fabricación de productos metálicos que representan el 6.6 por ciento del total de empresas con IED en el Estado. Por lo que respecta al comercio sobresale el comercio al por mayor de productos no alimenticios con más del 21.3 por ciento de empresas y el comercio de productos alimenticios al por mayor con el 2.7 por ciento dentro del sector comercial (Cuadro 3).

\section{Cuadro 3 - Distribución sectorial de las empresas con inversión extranjera en el Estado de Jalisco $2006^{(*)}$}

\begin{tabular}{|l|c|}
\hline \multicolumn{1}{|c|}{ Sectores } & $\begin{array}{c}\text { Participación \% con respecto al número de } \\
\text { establecimientos }\end{array}$ \\
\hline Agropecuario & 0.7 \\
\hline Minería y extracción & 1.8 \\
\hline Industria manufacturera & $\mathbf{2 6 . 5}$ \\
\hline Electricidad y agua & 0.1 \\
\hline Construcción & 2.2 \\
\hline Comercio & $\mathbf{2 5 . 6}$ \\
\hline Transportes y comunicaciones & 0.9 \\
\hline Servicios financieros & 14.6 \\
\hline Otros servicios ${ }^{(1)}$ & 27.6 \\
\hline TOTAL & 100.0 \\
\hline
\end{tabular}

(*)Al mes de septiembre de 2006

FUENTE: Elaboración propia con base en la Secretaria de Economía. Dirección General de Inversión Extranjera.

(1) Servicios comunales y sociales; hoteles y restaurantes; profesionales, técnicos y personales. Incluye los servicios a la agricultura, ganadería, construcción, transportes, financieros y comercio.

\section{Las tiendas de autoservicio el caso de Wal Mart}

Wal Mart es la cadena comercial de tiendas de autoservicios minorista más importante de México (Cuadro 4), y del mundo. De 1998 a la fecha presenta un crecimiento del 327 por ciento en el número de establecimientos y del 117 por ciento en cuanto a su cobertura geográfica. Actualmente cuenta con 901 establecimientos distribuidos en 141 ciudades del país (WAL MART, 2007b).

Es la tercera empresa mas importante que cotiza en la Bolsa Mexicana de Valores, superada únicamente por América Móvil y Teléfonos de México que juntas apenas alcanzan a representar los empleados que representa Wal Mart, más de 100 000. Sus ventas anuales son de 165000 millones de pesos, vende mas que Gigante, Soriana y Comercial Mexicana juntas. Incluye tiendas de autoservicio, tiendas de ropa y restaurantes, cotiza en la bolsa de valores y cuenta con rendimientos acumulados de $96.6 \%$ reinvirtiendo los dividendos en los 10 últimos años. 
Proporciona empleo a cerca de la mitad de los trabajadores del sector y obtiene el mayor porcentaje de las ganancias del comercio a detalle que se realiza en México, seguida muy por debajo de Comercial Mexicana y Gigante.

Cuadro 4 - Participación porcentual del empleo y ventas de las tiendas de comercio minoristas en México, 2005

\begin{tabular}{|l|r|r|}
\hline \multicolumn{1}{|c|}{ Auto Servicios } & $\begin{array}{c}\text { Empleo 2005 } \\
\text { (porcentajes) }\end{array}$ & $\begin{array}{c}\text { Ventas 2005 } \\
\text { (Porcentajes) }\end{array}$ \\
\hline Wal Mart de México & 42,92 & 37,83 \\
\hline Bodega Aurrerá & nd & 11,65 \\
\hline Wal Mart Supercenter & nd & 10,20 \\
\hline TOTAL WAL MART & $\mathbf{4 2 , 9 2}$ & $\mathbf{5 9 , 6 9}$ \\
\hline Grupo Gigante & 1,12 & 7,12 \\
\hline Súper Gigante & 1,66 & 1,34 \\
\hline Hipermercados Gigante & 4,31 & 2,93 \\
\hline Bodega Gigante & 1,63 & 1,31 \\
\hline Súper Maz & nd & $\mathbf{1 3 , 0 9}$ \\
\hline TOTAL GIGANTE & $\mathbf{1 8 , 7 5}$ & 11,09 \\
\hline Organización Soriana & 18,30 & $\mathbf{1 1 , 0 9}$ \\
\hline TOTAL SORIANA & $\mathbf{1 8 , 3 0}$ & 9,24 \\
\hline Controladora Comercial Mexicana & 12,37 & 2,75 \\
\hline Tiendas Comercial Mexicana & 3,19 & 2,91 \\
\hline Hipermercados Mega & 3,02 & 1,23 \\
\hline Bodega CM & 1,45 & $\mathbf{1 6 , 1 3}$ \\
\hline TOTAL COMERCIAL MEXICANA & $\mathbf{2 0 , 0 3}$ & 100,00 \\
\hline Total & 100,00 & \\
\hline
\end{tabular}

FUENTE: Revista Expansión, 2006.

Sam Walton su fundador, constituyó la empresa a finales de los años 60’s y al principio se concentró en mercados pequeños, en su mayor parte del sur de Estados Unidos. En 1969 contaba con 33 unidades en Estados Unidos. Al establecer posiciones cuasi monopólicas en estos mercados, la empresa fue muy rentable y tuvo capacidad para financiar una rápida expansión (VANCE, SCOTT, 1997. p. 11-67), a medida que la compañía se expandía, fue teniendo economías de escala, en términos de su capacidad para distribuir bienes a sus ubicaciones y en términos de su capacidad para negociar mejores precios con los proveedores (NICHOLSON, 2005. p. 408), Fue hasta que la compañía tenía una posición sólida en los mercados pequeños que empezó a incursionar en mercados más competitivos en zonas suburbanas del sur de los Estados Unidos. 
La estrategia de introducción de las tiendas Wal Mart en México, fue contrario a lo que se presentó en Estados Unidos. Se enfocó principalmente hacia las zonas metropolitanas y grandes ciudades en los estados más poblados (Mapa 1). El 40 por ciento de los establecimientos se localizan en el Distrito federal y su zona conurbada. Le sigue la zona centro ${ }^{(11)} \mathrm{con}$ el 30 por ciento. En estas dos regiones habita más del 40 por ciento de la población nacional.

\section{Mapa 1 - Expansión de las ciudades donde se ubican los negocios del corporativo Wal Mart en la República Mexicana 1999-2006}
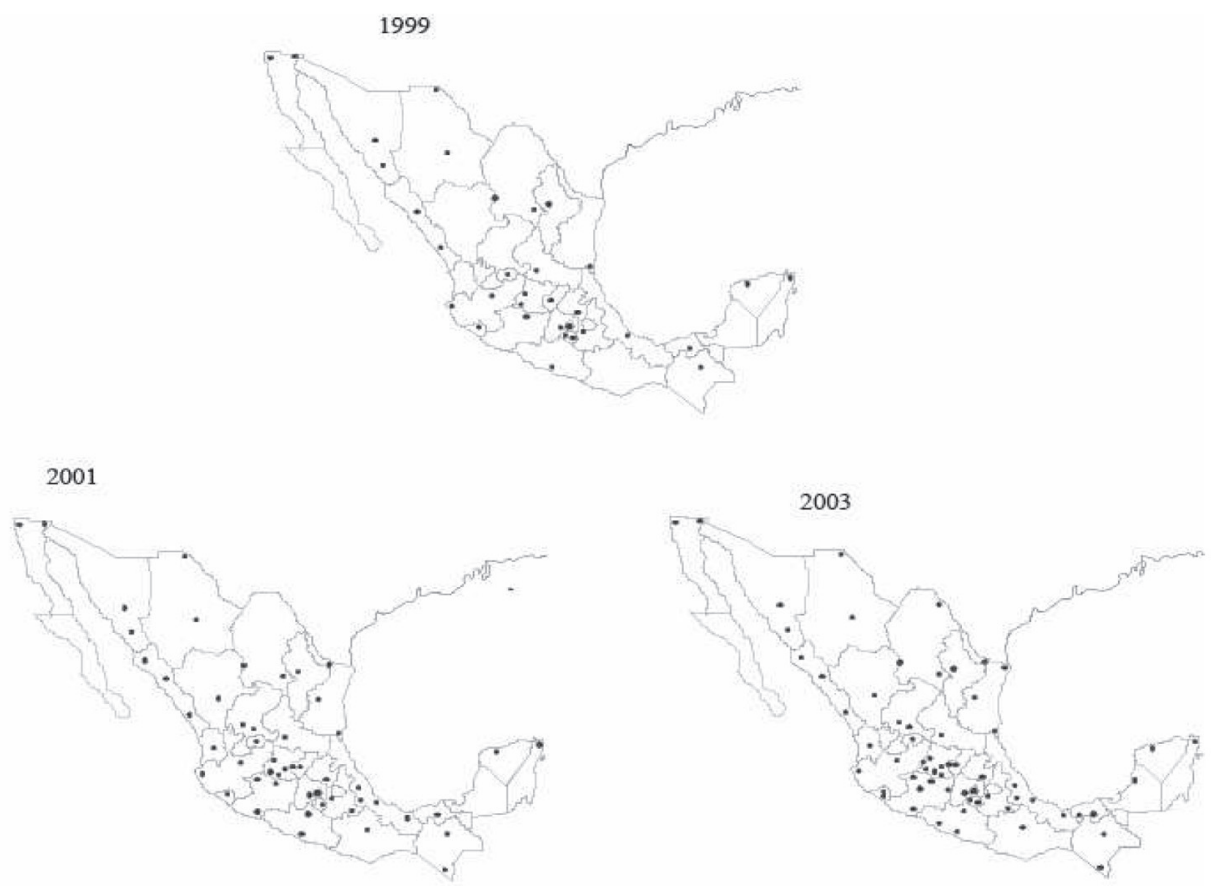

2005

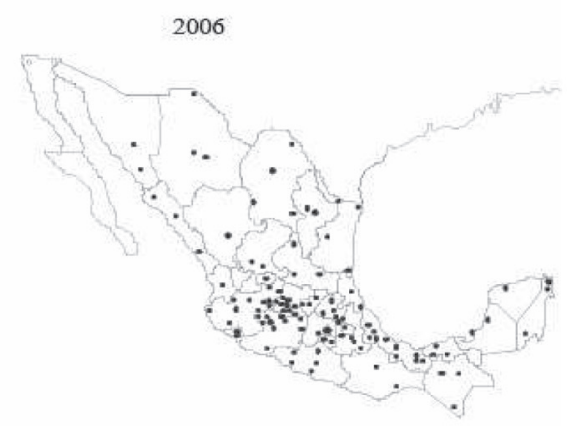

Fuente: Elaboración propia con base en los informes de Wal Mart de los años respectivos

(11) Abarca los estados de Aguascalientes, Colima, Estado de México, Guanajuato, Jalisco, Hidalgo, Michoacán, Morelos, Puebla, Querétaro, San Luis Potosí y Tlaxcala. 


\section{Contexto actual del mercado de tiendas de aUtoservicio en la ZMG}

La consolidación y expansión de las tiendas de autoservicio, iniciada en la ZMG desde la década de los noventa, coincide con la implementación y apertura de reformas de tipo estructural como son: Las desregulaciones comerciales y laborales, la apertura comercial (Tratados comerciales) y el debilitamiento del sindicalismo.

En los años 90 la metrópoli se vio impactada por una serie de acontecimientos económicos, productivos y políticos que le dieron un nuevo giro a la función económica que la ZMG había desempeñado hasta los años 80's. Se observaron una reducción de sus actividades productivas; presencia de la Inversión Extranjera directa enfocada hacia el sector comercial y a la manufactura y ensamble como parte complementaria de la industria electrónica; proliferación de plazas comerciales y aperturas de nuevos formatos de comercio como son los hipermercados y tiendas de conveniencia. La actividad económica ha influido sobremanera en la expansión urbana en ese mismo periodo la mancha urbana tuvo una expansión de 10.000 hectáreas pasó de 29.000 en 1990 a 39.000 en el año 2000 (Plan de Desarrollo Urbano y Vivienda, 2000). La transformación del territorio metropolitano obedeció a las nuevas dinámicas y consolidación de la producción global, del comercio y las finanzas (POZOS, 2004, p. 136; RODRÍGUEZ BAUTISTA, 2006).

La aparición de centros comerciales que iniciaron en Guadalajara a fines de la década de los sesentas atrajeron otras actividades y crearon zonas habitacionales. Ahora buscan mercados cautivos como zonas residenciales que puedan facilitar el desarrollo comercial.

La existencia de más de sesenta tiendas de autoservicio se explica por la preferencia de los consumidores tanto regionales como locales ${ }^{(12)}$. Guadalajara es el único punto a nivel nacional en donde convergen las cuatro grandes cadenas comerciales de tiendas de autoservicio. En la Ciudad de México no existe la tienda Soriana y en Monterrey la tienda Comercial Mexicana.

La preeminencia en este tipo de tiendas, la tienen Gigante y Wal Mart son las más representativas en cuanto al número de establecimientos (Cuadro 5).

Cuadro 5 - Tiendas de autoservicios ubicadas en la Zona Metropolitana de Guadalajara, 2007

\begin{tabular}{|l|c|}
\hline \multicolumn{1}{|c|}{ AUTOSERVICIO } & Porcentaje de tiendas respecto al total \\
\hline CHEDRAUI & 3.2 \\
\hline SORIANA & 7.9 \\
\hline COMERCIAL MEXICANA & 3.2 \\
\hline GIGANTE & 42.9 \\
\hline WAL-MART ${ }^{(*)}$ & 42.9 \\
\hline
\end{tabular}

FUENTE: Elaboración propia con base en los portales oficiales de la tiendas.

(*) Incluye El Corporativo Wal Mart, Superama, Sams Club y Bodega Aurrerá

(12) Se observa una práctica común en los patrones de compra en la ZMG con los consumidores regionales. Población de municipios fuera de la ciudad de Guadalajara acuden los fines de semana a abastecerse de mercancía de almacenes comerciales como son el caso de Sam's Club, Price Club. Ya sea para consumo personal o para la venta en sus pequeños negocios. 
Los establecimientos detallistas están interesados en proyectar una imagen específica hacia el consumidor con base en su estrategia comercial. Cada una de las tiendas goza de una identidad o personalidad que el cliente percibe, relaciona y recuerda.

El mercado tradicional en la ciudad así como el mercado de abastos está pasando por una crisis. Las bodegas ya no representan la función que antaño desempeñaban de ser concentradoras y distribuidoras de productos hacia el mercado de la región occidente y noroeste del país. Han venido a ser relevados por los grandes almacenes comerciales.

El capital comercial de origen internacional se empieza a materializar en la ciudad a mediados de la década de los noventa. La apertura de la primera tienda Wal Mart se inauguró en la ciudad en 1994, en Av. Vallarta y Rafael Sanzio.

Entre las tiendas departamentales de la cadena Wal Mart, ha establecido alianzas estratégicas con Aurrerá, restaurantes y almacenes de Ropa.

Bodega Aurora tiene la mayor contribución cerca de una tercera parte del total de ventas (Cuadro 6).

\section{Cuadro 6 - Unidades y participación porcentual de ventas del grupo Wal Mart a nivel nacional 2006}

\begin{tabular}{|l|c|c|}
\hline & $\begin{array}{c}\text { Participación } \\
\text { porcentual de las } \\
\text { unidades (\%) }\end{array}$ & $\begin{array}{c}\text { Contribución } \\
\text { de ventas (\%) }\end{array}$ \\
\hline Bodega Aurrerá (Tienda autoservicio) & $\mathbf{2 9 . 1}$ & $\mathbf{3 2}$ \\
\hline Sam's Club (Hipermercado) & $\mathbf{8 . 7}$ & $\mathbf{2 8}$ \\
\hline Wal Mart (Tienda de autoservicio) & $\mathbf{1 3 . 1}$ & $\mathbf{2 7}$ \\
\hline Superama (Tienda de autoservicio) & 6.8 & 5 \\
\hline Suburbia (Tienda de ropa) & 7.0 & 5 \\
\hline Vips (Restaurantes) & 35.4 & 3 \\
\hline Total & 100 & 100 \\
\hline
\end{tabular}

FUENTE: Datos de los Informes de Wal Mart, feb. 2007.

La zona metropolitana de Guadalajara no está ajena al fenómeno Wal Mart, tiene presencia en las tiendas de autoservicio del corporativo (Cuadro 7), aunque cada vez más se observa un desplazamiento hacia ciudades de menor tamaño del Estado como son: Tequila, Ameca, Lagos de Moreno, Jalostotitlán entre otros, que albergan poblaciones de 50.000 habitantes. 
Los Grandes Almacenes de Autoservicio ante la Globalización: El Caso de las Tiendas del Corporativo Wal Mart de Jalisco

Cuado 7 - Tiendas de Autoservicio que pertenecen al corporativo Wal Mart

\begin{tabular}{|c|c|c|}
\hline Nombre del Establecimiento & $\begin{array}{l}\text { Pisos de veta } \\
\text { (porcentajes) }\end{array}$ & $\begin{array}{c}\text { Participación de la ZMG a } \\
\text { nivel nacional }\end{array}$ \\
\hline Bodega Aurrerá & 18,5 & 4,2 \\
\hline Sam`s Club & 34,2 & 5,1 \\
\hline Wal Mart & 39,6 & $\overline{7,6}$ \\
\hline Superama & 7,7 & $\overline{4,9}$ \\
\hline Total & 100,0 & 5,2 \\
\hline
\end{tabular}

FUENTE: Elaboración propia con base en los Informes de la empresa Wal Mart, fev. 2007.

La percepción del consumidor de los comercios detallistas, es lo que se define como la imagen del detallista. Imagen sobre la cual depende la decisión de compra en determinada tienda o en otra. El consumidor acudirá a la tienda que sea más de su agrado. De acuerdo a Nicholson (2005, p. 408) la ventaja de las tiendas minoristas, en este caso de Wal Mart, alcanzó su posición explotando economías de escala y también las ventajas que obtuvo por ser la primera en jugar con su estrategia inicial de trabajar en pequeñas localidades en los Estados Unidos. A diferencia de lo experimentó en México, se ubicó principalmente en las grandes ciudades

Se prevé que el crecimiento acelerado de Wal Mart continúe. México es uno de los principales países que presenta el mayor aceleramiento en la introducción de tiendas Wal Mart. Debido a que es un mercado potencial, cuenta con más de 100 millones de habitantes, de los cuales 50\% tiene menos de 21 años y $33 \%$ menos de 14 años de acuerdo a las cifras del último conteo de población.

\section{El comportamiento estratégico de Wal Mart}

La estrategia de multiformato hasta el momento le ha funcionado mayormente a Wal Mart. En la introducción de un producto nuevo y la devolución del dinero, con su introducción podría explicar algunas de las primeras ventajas de ser la primera en jugar, una razón más importante tal vez, se debe a los problemas de información que aportan los consumidores, porque estos no saben si cumplirá con lo que supuestamente debe proporcionar el producto nuevo. Por tanto, alguien que compra el producto se expone a ese riesgo. Si el producto funciona como es anunciado, el consumidor podría decidir quedarse con él mientras se van desarrollando otras marcas "copiadoras". Por lo tanto, ser el primero tiene sus ventajas. Por eso Wal Mart ofrece el $200 \%$ cuando un producto de introducción no reúne las expectativas del cliente (NICHOLSON, 2005, p. 408).

La característica principal de los establecimientos comerciales se basa en sus altos niveles competitivos que se observan en la presentación, precio, calidad y diversidad de los productos así como en la organización de la empresa, utilización de la mercadotecnia, tecnología aplicada al control, producción, distribución y comercialización de las mercancías, 
publicidad a través de diversos medios de comunicación como son: radio, televisión, folletos e internet. Todas estas herramientas se van adecuando con base en el comportamiento de la competencia (Cuadro 8).

\section{Cuadro 8 - Wal Mart estrategias de mercadotecnia}

\begin{tabular}{|l|}
\hline Alianzas con empresas líderes del mercado \\
\hline Precios bajos todos los días y por tiempo determinado se bajan aún más. \\
\hline Prácticas monopolísticas \\
\hline Adquisición de otras cadenas de supermercados \\
\hline Satisfacción $200 \%$ (") \\
\hline Liquidación \\
\hline Superartículo, es un articulo diseñado exclusivamente para el cliente \\
\hline Marcas exclusivas en salud y belleza "equale" \\
\hline Marcas exclusivas en abarrotes y químicos "Great value" \\
\hline Empleados siempre disponibles para los clientes \\
\hline Pasillo amplios \\
\hline Recibe vales del gobierno \\
\hline Pagos de servicios diversos (tenencia, agua, teléfono) \\
\hline Crédito al instante \\
\hline Servicios de apartado \\
\hline Money Gram \\
\hline
\end{tabular}

FUENTE: Elaboración propia con base los informes anuales y promociónales de la tienda.

(*) Significa que si el producto adquirido no es de entera satisfacción se lo cambian por otro y además le devuelven su dinero. Aplica solamente en los artículos que por primera vez se exhiben en piso de ventas.

La política de Wal Mart precios bajos todos los días, se analizaron los precios de artículos de la canasta básica y artículos de lujo de las tiendas Wal Mart y Gigante en septiembre de 2006. Llegando a la conclusión de que sus cercanos competidores Gigante, Comercial Mexicana y Soriana son seguidores de los precios de Wal Mart. Las diferencias mayores de precios se dan en los artículos de la canasta básica hasta un 32\% (COTA YAÑEZ, 2006) es más barato comprar en Wal Mart que en Gigante. De igual forma Álvarez y Tilly (2006, p. 956) encentran que entre Wal Mart y Gigante hay una diferencia del 7\% en cuanto al total de productos con precios bajos.

Aun y cuando la diferencia entre los productos de las diferentes tiendas de comercio minorista sea de centavos a favor de Wal Mart la gente se deja influenciar por la ilusión del precio, además de que al acudir al establecimiento compra otro tipo de artículos que no necesita y al final gasta lo no planeado. 
Dentro de las estrategias competitivas de Wal Mart figura el precio y la ubicación estratégica de sus negocios (Mapa 2).

En un trabajo anterior en la ZMG (COTA, ZARZOSA, 2007) se observó un cambio en el patrón de compras de los habitantes. Existe una marcada preferencia de la población a hacer sus compras en las tiendas de autoservicio. Wal Mart fue mencionado en el 50 por ciento de los casos, seguido de la tienda de autoservio denominada Gigante.

El modelo de oligopolio de Hotelling habla de la competencia por el espacio e indica que conforme los productores se acerquen más al consumidor, se generarán economías de escala pero también pueden llegar a generarse aglomeraciones (NICHOLSON, 2005, p. 393). La economía de escala se da cuando un consumidor desea adquirir un bien o servicio se desplazará hasta los lugares donde podrá contar con una variedad de posibilidades de elección sin tener que hacer grandes costos en sus desplazamientos. Al respecto también Nicholson (2005, p. 377) plantea el modelo por la competencia por espacio, los consumidores están ubicados uniformemente en un circulo, que puede presentar un espacio geográfico real o el espacio de un producto, la presencia de dos empresas altamente competitivas en los mismos productos, en este caso, Wal Mart y Gigante (A y B), desalienta la entrada de otra (C). En el mapa se observan algunas zonas en las que el corporativo Wal Mart se ubica.

Se observa que existen puntos muy específicos y diferenciados de este tipo de tiendas. Para el caso de Wal Mart, observamos que existe un corredor en la ubicación, específicamente en las principales avenidas y cerca de Plazas comerciales y fraccionamientos privados de alta plusvalía como lo son Puerta de Hierro, ciudad Bugambilias.

Las tiendas Wal Mart presentan un patrón de ubicación cual si fuera la columna vertebral de la ciudad, la atraviesan de suroeste a noreste. La mayoría de los establecimientos se ubican en la zona poniente de la ciudad que abarca principalmente el municipio de Guadalajara y Zapopan y es donde habita la población de mayores ingresos.

Se puede observar que las tiendas de autoservicios han sacado provecho de las economías de escala generadas por la concentración. En un principio Gigante se aprovechó de esta estrategia al ubicarse en las principales plazas comerciales y fue considerada como tienda ancla ${ }^{(13)}$, ahora, se detecta que Wal Mart busca competir por medio de la localización estratégica de sus negocios. 
Mapa 2. Ubicación de las tiendas de autoservicio del Corporativo Wal Mart en la ZMG 2007

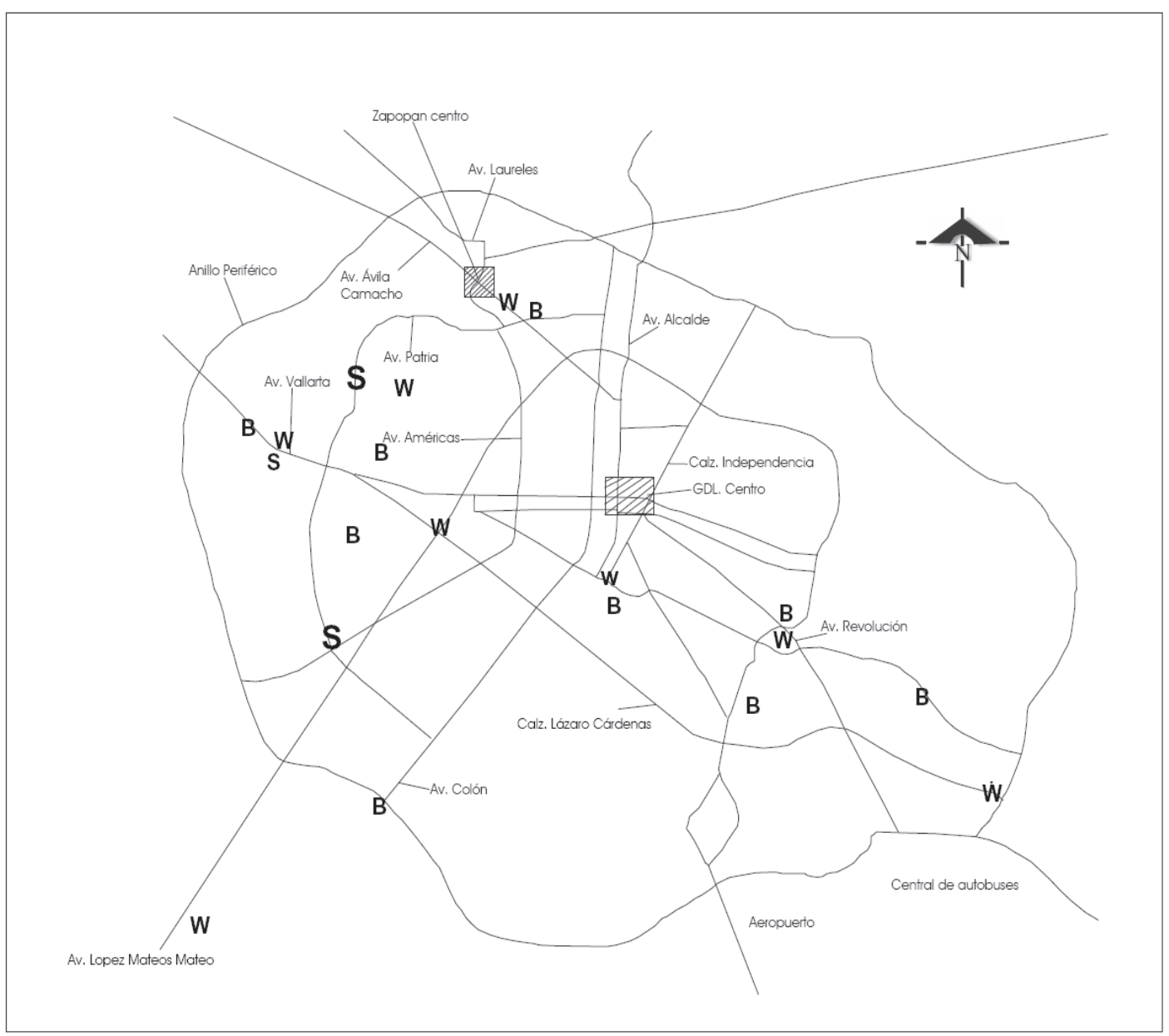

Fuente: Elaboración propia con base en el directorio de Tiendas de Autoservicio del Corporativo Wal Mart 2007.

La ubicación del corporativo Wal Mart en la ciudad ha propiciado cambios en la infraestructura urbana. Se están creando centros económicos que aprovechan las economías de aglomeración al atraer a actividades complementarias como son: restaurantes, locales de comida rápida, autolavados, neverías entre otros.

La creación de los centros económicos ha requerido de modificaciones en la estructura urbana de la ciudad, mediante la creación de pasos a desnivel, remozamiento de banquetas, alumbrado. La imagen urbana ha adquirido una nueva forma y función.

(13) Se considera tienda ancla, a los establecimientos a los que la gente tiene que acudir a hacer sus compras más necesarias. Por lo regular las tiendas de autoservicios fueron consideradas así, cuando se instalaron en las principales plazas comerciales de la ciudad. 


\section{Conclusiones}

Se observan cambios en la distribución del comercio minorista. Estructura de la actividad, patrones productivos, tecnológicos y organizacionales de la producción, formas de intermediación en los transportes, novedosos canales de comercialización, organización del espacio urbano, cambios culturales, hacia una atención personalizada del cliente, padrón de compras menos frecuente pero de mayor volumen.

En México se observa un incremento del comercio minorista en las grandes ciudades. A diferencia de cómo inició la conquista del mercado en los Estados Unidos. Actualmente está empezando una progresiva expansión hacia las ciudades medias del estado de Jalisco, compitiendo por medio del formato de Tienda denominado Bodega Aurrerá que está enfocado hacia consumidores de ingresos menores.

Las tiendas de comercio minorista en México se encuentran inmersas dentro de un mercado oligopolista para la venta y podría decirse que mantienen características de monopsonio con sus proveedores, para el caso de Wal Mart, por las grandes cantidades de compra que realiza.

Las grandes cadenas de autoservicio que tan sólo representan el 0.2 por ciento de los 562 mil establecimientos comerciales del país son las que determinan los precios de los artículos de consumo generalizado en los principales centros urbanos.

La apertura comercial ha propiciado e incentivado la proliferación de capitales extranjeros dentro de las inversiones de las tiendas de autoservicios.

Los competidores se han tenido que ir ajustando a las políticas de comercialización de Wal Mart, ello ha traído como consecuencia el cierre de pequeños establecimientos que no han podido competir. Así como de algunas tiendas de autoservicio ya consolidadas como Gigante.

La estrategia de competencia de Wal Mart se basa en precios, productos, publicidad y ubicación.

La estructura urbana se ha modificado para dar cabida a un nuevo modelo de paisaje urbano en las ciudades mediante la creación de centros comerciales.

\section{REFERENCIAS BIBLIOGRÁFICAS}

BASKER, Emek. Job creation or destruction? Labor-merket effects of Wal Mart expansion. Review of Economics and Statistics, v. 87, n. 1, p. 174-183, 2005.

BAUTISTA, Juan Jorge Rodríguez. La nueva economía y sus efectos en la ciudad de Guadalajara. La última década del siglo XX. Universidad de Guadalajara, 2006.

CABRALES, B. Felipe (comp.). Espacio urbano, cambio social y geografía aplicada. Jalisco: UdG, 1993.

GALVÁN, José Luis Álvarez; CHRIS, Tilly. Participación extranjera en las tiendas de autoservicio en México: el efecto de Wal Mart. Comercio Exterior, México: Bancomext, v 56, n. 11, nov. 2006.

HORSKY, Dan; NELSON, Paul. New brand positioning and pricing in an Oligopolic Market. Marketing Science, v. 11, n. 2, p. 133-153, 1992. 
NICHOLSON, Walter. Microeconomía intermedia. 9. ed. México: Thompson, 2005.

PLAN DE DESARROLLO URBANO Y VIVIENDA. Jalisco, 2000.

PONCE, Fernando Pozos. Guadalajara: ¿en búsqueda de una nueva función urbana? Revista Espiral, Universidad de Guadalajara, v. X, n. 29, p. 135-160, ene./abr. 2004.

PORTER, Michael. Towards a dynamic theory of strategy. Strategic Managerial Journal, v. 12, p. 95-117, 1991.

REVISTA EXPASIÓN. Las 500 empresas más importantes de México. México, v. 946, 28 jun. 2006.

SISTEMA ESTATAL DE INFORMACIÓN JALISCO. SEIJAL. Gobierno del Estado de Jalisco, 2007.

VANCE, Sandra; SCOTT, Roy. Wal Mart. Una historia del fenómeno de Ratail de Sam Walton. Argentina: Temas, 1997.

YÁNEZ, Rosario Cota; CODOCERO, Alejandra Zarzosa. El impacto de la reestructuración de las tiendas de Conveniencia en la Zona Metropolitana de Guadalajara. Ponencia de la Asociación Latino-americana de Sociología. Guadalajara, p. 14-18, ago. 2007.

YAÑEZ, Rosario Cota; COTA, Brenda. Estrategias competitivas: el caso de Wal Mart de la Zona Metropolitana de Guadalajara. Ponencia presentada en el Encuentro Nacional sobre Desarrollo Regional en México. Mérida, México, nov. 2006.

WAL MART. Disponible en: <http://www.walmartmexico.com.mx/walmex_BMV.pdf> Acesso em: 15.3.2007.

Disponible en: <http://www.walmartmexico.com.mx/informe2004/informe_esprenta bilidad.html > Acesso em: 15.3.2007. 